\title{
Gestión educativa y calidad de formación profesional en el Instituto Superior Pedagógico "Gran Pajatén”
}

\author{
Carlos Miguel Aguilar Saldaña \\ Agua0122@hotmail.com \\ Carol Beatriz Bao Ratzemberg \\ tptbao@hotmail.com \\ Gabriela Gabriela del Pilar Palomino Alvarado \\ gabpal11@hotmail.com \\ Karl Whittembury García \\ Karlwg1@gmail.com \\ Héctor Manuel Suarez Ríos \\ msuarez2004@hotmail.com \\ Renán Saavedra Sandoval \\ renan.saavedra@hotmail.com \\ Jimmy Pacherrez Riva \\ rivapm@ hotmail.com \\ Escuela de post grado \\ Universidad César Vallejo.
}

\section{RESUMEN}

Este trabajo se caracteriza principalmente por determinar relación de la Gestión Educativa, y la Calidad de Formación Docente. La justificación coincide con las afirmaciones de Sorados Palacios, M. (2010) que la calidad de la gestión de una institución educativa, es resultado del rendimiento académico, cooperación de los docentes, cumplimiento de la programación curricular, participación de los padres de familia y asignación de recursos. El objetivo es analizar la Gestión Educativa para determinar su relación con la Calidad de Formación Profesional docentes que forman en la institución. Se recurrió al método lógico deductivo y al diseño de investigación no experimental transversal correlacional. Como técnicas de recolección de datos se ha empleado ficha de observación, para documentos académicos y administrativos; las libretas de notas, para registrar las actividades más relevantes; la encuesta por cuestionario se ha empleado para evaluar el proceso de formación profesional en los alumnos de la Especialidad de Lengua y Literatura; y la entrevista no estructurada en ciertas circunstancias. Por último, como resultado se obtuvo que existe una relación alta y significativa entre la Gestión Educativa de nivel regular y la Calidad de Formación Profesional, con una correlación de 73,1\%.

Palabras clave: gestión; educación; calidad de la educación; formación docente. 


\title{
Educational management and quality of professional teacher training.
}

\begin{abstract}
:
This work is mainly characterized by determining the relationship between Educational Management and the Quality of Teacher Training. The justification coincides with the statements of Sorados Palacios, M. (2010) that the quality of the management of an educational institution is the result of academic performance, cooperation of teachers, compliance with the curricular programming, participation of parents and resource allocation. The objective is to analyze Educational Management to determine its relationship with the Quality of Professional Training of teachers who train in the institution. The deductive logic method and the correlational cross-sectional nonexperimental research design were used. As data collection techniques, observation files have been used for academic and administrative documents; notebooks, to record the most relevant activities; The survey by questionnaire has been used to evaluate the professional training process in the students of the Language and Literature Specialty; and the unstructured interview in certain circumstances. Finally, as a result, it was obtained that there is a high and significant relationship between Educational Management at a regular level and the Quality of Professional Training, with a correlation of $73.1 \%$.
\end{abstract}

Keywords: management; education; Quality of education; teacher training.

Artículo recibido: 03. agosto. 2020 Aceptado para publicación: 09. setiembre. 2020

Correspondencia: agua0122@hotmail.com Conflictos de Interés: Ninguna que declarar 


\section{INTRODUCCIÓN}

La educación es parte de nuestra vida cotidiana y fuerza de motor para el desarrollo y crecimiento del país. En tal sentido la gestión educativa aparece como eje estratégico, decisiva y valiosa para la conducción y dirección de las instituciones educativas, de aquí parte la importancia en esta época contemporánea, por ello constituir la clave fundamental y herramienta básica para alcanzar el desarrollo y calidad de la educación peruana, especialmente en la educación superior.

En la actualidad, han nacido nuevos enfoques y formas de ver la educación con miras a una educación de calidad. La descentralización de la gestión de la educación en el Perú se viene realizando hace varios años y que hasta ahora no llega a plasmarse en una completa transferencia de funciones administrativa y económica. Lo que se busca es contribuir a mejorar la calidad de los prestadores de los servicios de educación superior a través de una correcta y oportuna retroalimentación, para una educación permanente de calidad e informar a la dirección y el equipo de gestión para tomar las medidas correctivas necesarias para dirigir la gestión de Gestión Educativa con calidad.

Frente a lo expuesto aparecen los Fundamentos de la Administración científica (Taylor, 1961).La necesidad de una organización científica, en lugar de la vigilancia suspicaz y la guerra más o menos encubierta que caracteriza el tipo ordinario de administración, existe una cooperación cordial entre la dirección y los obreros. Desde esta perspectiva, la Institución Educativa será un sistema netamente autoritario, donde las reglas, las normas, las indicaciones y las responsabilidades guían el comportamiento de las personas, para lo cual, cada uno de los integrantes del sistema, previamente, sabrá cuál es su rol dentro de la organización y cuáles son sus compromisos y tareas a cumplir con el fin de lograr eficiencia en una estructura racionalizada.

Conceptualiza a la Gestión Educativa (Alvarado Oyarce.1998) La gestión se entiende como el sistema de actividades estratégicas y planificadas que se realizan guiadas por procedimientos y técnicas adecuadas para lograr determinados fines o metas. Estos fines, metas o propósitos, pueden ser perseguidos por personas, instituciones o empresas, tanto públicas como privadas. La gestión educativa puede definirse como el conjunto de actividades y diligencias estratégicas guiadas por procedimientos y técnicas adecuadas para facilitar que las instituciones educativas logren sus metas, objetivos y fines educacionales.

Las teorías de la Gestión Administrativa y organizacionales, permiten comprender y entender a las organizaciones, las cuales, son complejas, dinámicas, con capacidad de aprendizaje, conflictivas, son sistemas abiertos, con estructuras jerárquicas determinadas, con relaciones organizacionales permeadas por el poder, el control, el liderazgo, la división del trabajo, entre 
otras; (Dávila, Carlos. 2001,) define las organizaciones como ente social, creado intencionalmente para el logro de determinados objetivos mediante el trabajo humano y recursos materiales; por su parte, la administración es la acción específica que realiza el sujeto dentro de la organización para lograr los objetivos propuestos, apoyado en el proceso administrativo (planear, organizar, dirigir, coordinar y controlar).

En la actualidad y desde los últimos cinco años, con la incorporación de los modelos de Gestión de Calidad, la necesidad de focalizar las políticas en los resultados de aprendizaje de los estudiantes y los aportes de la teoría educativa, especialmente de los movimientos de escuelas efectivas y eficacia escolar; se ha tomado conciencia real del potencial que tiene el desarrollo de una gestión efectivamente descentralizada y el rol principal de la dirección en el logro de resultados. El desafío que enfrentan los colegios, para avanzar a grados mayores de calidad en el servicio educativo, es transformarse en Instituciones que se auto gestionan.

La toma de decisiones de manera endógena permite a los centros escolares, primero determinar su rumbo según su realidad local y, por otro, asumir responsabilidades por los resultados de su gestión. La falta de capacidad de gestión del currículo no permite, en muchos casos, aprovechar recursos valiosos que se pone a disposición de los Centros Educativos. En este sentido, diferentes programas han estimulado en las escuelas la creación de Equipos de Gestión, conformado por el director(a), profesores y en algunos casos por padres y alumnos como parte de un proceso de planificación estratégica con el fin de desarrollar su proyecto educativo institucional acorde a la realidad de sus alumnos (PEI). A nivel del Ministerio de Educación, se ha creado para la dirección escolar, el Marco para la Buena Dirección. (Minedu 2017), que constituye un estándar para el mejoramiento del desempeño directivo y la posibilidad de ampliar la autonomía de los directivos en el ejercicio de la gestión. Uno de los ejes centrales de esta iniciativa es la Gestión Curricular como elemento preponderante en los procesos de mejora.

El Enfoque del Desarrollo de la Gestión Educativa se caracteriza por considerar en el desarrollo del proceso educativo a los procesos de gestión, tales como planificación, organización, dirección y control. El desarrollo adecuado de cada uno de estos procesos tiene como propósito lograr los objetivos educacionales previstos. El Enfoque sistémico de la gestión educativa, puede definirse como el conjunto global y unitario de elementos que interactúan dinámicamente para lograr determinados propósitos. Por ejemplo en un Ministerio hay personas (profesionales, técnicos y auxiliares) que interactúan, infraestructura y equipos tecnológicos, materiales, etc. Que en conjunto permiten alcanzar objetivos y propósitos previamente definidos. En opinión de Alvarado, Oyarce. El enfoque de sistemas como categoría analítica, permite por un lado la representación mental de un sistema mediante el uso de modelos para efecto de análisis del todo 
o de las partes en interacción con el análisis de sistemas, identificando problemas y posibles soluciones, a efectos de lograr el mantenimiento, la regulación o el desarrollo del propio sistema. El Enfoque Gerencial de la Gestión Educativa se caracteriza por considerar en el desarrollo del proceso educativo a los procesos de gestión, tales como planificación, organización, dirección y control. De igual forma, manifiesta que la planificación es el ordenamiento racional y sistemático de actividades y proyectos a desarrollar, asignando adecuadamente los recursos existentes, para lograr los objetivos educacionales .En la planificación los elementos más importantes que siempre se toman en cuenta son: actividades, recursos y estrategias, todos ellos orientados a lograr fines educacionales.

\section{Métodos para la planificación educativa}

Por su lado (Puelles, Manuel. y otros 1996), Expresan que los métodos utilizados en la planificación educativa son: El método de las previsiones de mano de obra (recursos humanos). Según este método la educación se orienta a la preparación de la mano de obra necesaria para el proceso productivo, mediante la planificación se busca satisfacer los requerimientos de las demandas sociales y las necesidades ocupacionales futuras, (educación y empleo). El método de la relación educación rendimiento tiene en cuenta el número de personas instruidas y el número de alumnos que terminan los estudios en los diversos grados, relacionados directamente con la producción nacional de bienes y servicios (enfoque eficientista). El Método de las cantidades globales establece una correlación entre las necesidades de enseñanza individual y social, con criterios extraídos de enfoques empíricos sobre la situación de la educación en otros países (educación comparada).

En la planificación educativa, según este enfoque los principios rectores constituyen los criterios y pilares fundamentales que guían la previsión y desarrollo de todo el trabajo de las instituciones educativas, que tiene como orientación y propósito lograr las metas y fines trazados en el plan de Desarrollo Educativa Institucional, en relación directa con los grandes objetivos nacionales y las aspiraciones sociales y económicas de la población. En el enfoque gerencial de la educación como aplicación de los principios y postulados de la planificación estratégica, (en las instituciones educativas) se trabajan con el PCC (Proyecto Curricular de Centro) y el PDI (Proyecto de Desarrollo Institucional). El PCC es entendido como instrumento dinámico y articulador de todas las actividades educativas que realizan docentes y alumnos, para lograr sus objetivos de aprendizaje y de desarrollo escolar y que a su vez sirve de guía o patrón de referencias para las decisiones didácticas específicas. El PCC es el medio o instrumento que permite a los enseñantes insertar su responsabilidad y su actuación didáctica dentro de un conjunto más amplio, 
posibilitando que la tarea personal en un aula, grupo o clase se articule constantemente en un marco más general, de cuya definición ha sido protagonista.

El Enfoque Gerencial es el PDI (Proyecto de Desarrollo Institucional), es un documento orientador producto de un proceso de reflexión colectiva que postula básicamente el perfil del centro educativo en términos de un patrón axiológico de los perfiles (alumnos y docentes) y enfoques pedagógicos pertinentes, así como los objetivos y estratégicos de desarrollo institucional a mediano y largo plazo. También, uno de los componentes claves que forman parte estratégica del enfoque gerencial de la gestión educativa es el Proceso de Organización. La organización como proceso que sigue a la planificación tiene como propósito, distribuir y dosificar racionalmente las actividades que se van a realizar, y asimismo designar responsabilidades y asignar los recursos de manera eficiente para lograr las metas lo más acertadamente posible.

Por su lado (Quinn, Rober. 2015). Manifiesta los Principios organizacionales. La organización como todo proceso orientado a facilitar la realización de metas mediante distribución de recursos, tanto humanos, como logísticos, también se guía por principios rectores. Según Kast, F. E. y Rosenzwelg, Y. E., los principios que orientan a toda organización son: La especialización organizacional y la división del trabajo, el de jerarquía, el de autoridad, responsabilidad y obligación, el de mano de obra, de control, el de Línea y Staff. Componentes organizacionales que se articulan e interactúan para facilitar el logro de las metas y objetivos de la organización son: los Organigramas, el Manual de Organización y Funciones, el Manual de Procedimientos Administrativos, y el Reglamento Interno. Otro de los elementos es el Proceso de Dirección. La dirección dentro del enfoque gerencial supone la conducción de la organización, basados en un conjunto de decisiones acertadas para promover la participación de todos los administrativos en la marcha exitosa de la empresa o institución.

Como señala (Thompson Ardicark. y Strickland.2015.) Una buena estrategia y su adecuada implantación son las señas más confiables de una buena dirección. Los principios que deben guiar la dirección de una institución son: Principio de amortización de metas. Según este principio, las metas de la institución educativa deben lograrse teniendo en cuenta las expectativas de todos los integrantes de la comunidad educativa, es decir, de los profesores, alumnos, padres de familia, administrativos, el gobierno, la población y la sociedad. Principio de compatibilización de objetivos. Según este principio las metas personales de los miembros de la institución deben estar en armonía con las metas institucionales, con el propósito de optimizar el éxito de la institución. Principio de motivación. 
El personal que labora en la institución sin duda, para un mejor desempeño de su función o labor requiere de una buena motivación, de ello se deduce que aquel que esté mejor motivado, trabajará y producirá mejor. Existe una dinámica de interacción entre la persona y su trabajo, que vendría determinada tanto por las demandas y recursos que plantea el trabajo, como por las demandas y recursos que la persona posee y que intenta satisfacer y aplicar en su trabajo. Cuando mayor sea el ajuste entre las características y objetivos del trabajo y de la persona, mayor será el ajuste entre las demandas y recursos que ofrece el trabajo y la persona, cuyo resultado sería de beneficio tanto personales y laborales, (Piero Jojan. y Prieto Ferdinand. 2015).

Hay que entender que el liderazgo es la capacidad que tiene el director para convencer, guiar, conducir y dirigir de manera eficaz y optima a los miembros de la institución, generando en ellos un sentimiento de lealtad y entrega total e incondicional sin necesidad del peso obligacional de los mismos. Liderazgo es la ciencia de conducir una organización de la situación actual a una situación futura, comparativamente mejor, donde las personas son el principal factor de cambio (Lepeley Miller. 2016).

El principio de las buenas decisiones orienta a la dirección, para que esta se haga, tomando decisiones adecuadas, acertadas y efectivas y para ello se requiere lógicamente que el director posea conocimientos científicos y técnicos necesarios y la experiencia gerencial suficiente. Las decisiones, para que sean adecuadas deben tener como resultado beneficios que favorezcan a la institución. Principio de la eficacia de la delegación. Es necesario y pertinente que el director dirija y conduzca la institución con la participación decidida de las personas de su confianza, delegando en ellos responsabilidades y atribuciones que considere conveniente y reservando para él, aquellas que solo pueda ejercerlas personalmente. La delegación de funciones y responsabilidades, es considerada sin duda, una acción estratégica muy necesaria para lograr las metas institucionales, por ello es pertinente tomar en cuenta ciertos elementos o factores que optimicen esta decisión. Principio de la supervisión directa. El director debe estar en contacto permanente con los administrados, de tal manera que le sea viable, observar, valorar y evaluar las funciones y responsabilidades que cumplen cada uno de ellos.

El principio de comunicación efectiva es fundamental en la convivencia institucional, en tal sentido, ella debe ser clara, precisa y oportuna, de tal manera que todos entiendan y comprendan las responsabilidades y funciones que les toca desempeñar. Principio de cambio continúo. El dinamismo, que debe caracterizar al director, y que conduce al cambio permanente de las condiciones físicas y tecnológicas de la institución es sumamente necesario, ya que de ello depende desarrollar una buena dirección para lograr las metas y fines que se persigue. El proceso de control. El control en la gestión educativa según el enfoque gerencial de la educación que 
consiste en evaluar, medir y ponderar los resultados de lo ejecutado, con lo previsto en el plan o programa de desarrollo de la Institución Educativa, pudiendo ejercer dicho control durante todo el proceso y al final del mismo.

El principio de control, nos dice (Quinn, Robert y Otros, 2016) que tiene cuatro pasos: El control en la gestión educativa. Todo programa de actividades, debidamente planificada requiere de un sistema de control. La gestión educativa con mayor razón, ya que de ésta depende el éxito en el logro de los resultados institucionales, para ello el Estado ha instituido dos sistemas: el sistema de supervisión educativa y la inspección educativa. Por control de calidad educativa debemos entender la acción de cautela y verificación sistemática del proceso educativo, con el fin de que el producto responda a las expectativas de la sociedad, o sea que satisfagan algunos estándares establecidos en términos de nivel de conocimientos, dominio de habilidades, practica de valores, actitudes positivas en relación a su persona, la familia, la comunidad, etc.

El sistema de supervisión educativa según (Alvear, Osvaldo. 2016). Manifiesta que fue concebida como capacitación en el trabajo, es decir, se evaluaba para detectar a tiempo las deficiencias y corregirlos oportunamente, sin embargo, en la actualidad ha perdido su verdadero sentido, convirtiéndose en una acción burocrática y reprimente para el docente y los administrados. La función preventiva fue uno de los atributos de la supervisión educativa, desde que fue implementada. El sistema de inspección educativa fue implementada conjuntamente con la supervisión educativa, pero a diferencia de esta, constituye una medida preventiva, el sistema de inspección fue diseñado para un control posterior a las actividades realizadas, con el propósito de comparar los resultados con las expectativas del plan o programa previamente diseñados y luego dado el caso, aplicar los correctivos correspondientes, incluyendo sanciones. La auditoría es una evaluación detallada y minuciosa que se realiza posterior a la ejecución de las actividades funcionales o administrativas, realizadas por funcionarios públicos y técnicos competentes, para luego emitir un informe con sus respectivas conclusiones.

En cuanto a la calidad de formación profesional. Formación profesional. Según el Diccionario de la Real Academia de la Lengua Española, profesión es facultad, arte u oficio, que posee una persona como resultado de una preparación formal. Teniendo en cuenta esta definición puntual podemos decir que formación profesional es la preparación y adiestramiento de una persona en oficios, facultades o artes para que las ejerza públicamente. En términos más rigurosos podemos decir que es la preparación y capacitación sistemática e institucionalizada del individuo en conocimientos, habilidades y actitudes por áreas y dominios, para el ejercicio de una determinada rama del saber, en correspondencia con las necesidades del mercado laboral. Las profesiones se 
caracterizan porque en ellas se incluye un conjunto de acciones que implican conocimiento, técnicas y algunas veces, una formación cultural científica y filosófica.

Según (Gonzales, Héctor. 2015). La formación profesional abarca todos los aspectos necesarios para garantizar un desempeño pleno y compatible con las aspiraciones, sociales de la población. Por ello cuando se refiere a que debe preparar al hombre en conocimientos, significa que las materias que conformen el área de formación general deben ser de dominio teórico pleno por parte del profesional; lo mismo debe serlo en la preparación y desarrollo de habilidades y destrezas. Y aquello que resulta trascendente en el hombre que ejerce una profesión, es sin duda, la actitud ética y moral que debe demostrar en todo su desenvolvimiento y ejercicio profesional. La enseñanza superior, como marco general de la formación profesional requiere de un conjunto de orientaciones pertinentes para convertirse en instrumento de desarrollo de los pueblos. En una sociedad, la enseñanza superior, es a su vez uno de los motores del desarrollo económico y uno de los polos de la educación a lo largo de la vida. Es, a un tiempo, depositaria y creadora de conocimientos. En un mundo en que los recursos cognoscitivos tendrán cada día más importancia que los recursos materiales como factores del desarrollo, aumentará forzosamente la importancia de la educación superior y de las instituciones dedicadas a ella.

Nos dice (Delors, J.1996). La Calidad de formación profesional. Podemos definir el concepto de calidad, como el conjunto de características, atributos, cualidades y propiedades de los bienes productos y bienes servicios, que le confieren la capacidad de satisfacer plenamente las necesidades, requerimientos y exigencias de los clientes, sean estos consumidores de un producto o usuarios de un servicio.

Específicamente, hablando de calidad de formación profesional, podemos decir que es el conjunto de requisitos y competencias que debe poseer el egresado de una carrera profesional, para satisfacer plenamente las expectativas y exigencias. El egresado de una entidad formadora se convierte en un producto que irremediablemente debe competir con otros, que posiblemente tengan mejores condiciones de formación profesional, es decir, de mejor calidad.

La calidad educativa, por su parte, (Domínguez, Gresty.1993) define como la conjunción de tres dimensiones o niveles. Que son los siguientes: La calidad normativa de la formación, La calidad de la formación a través de la adaptación de los contenidos a las necesidades y expectativas y motivaciones vitales y de formación de la comunidad educativa, La calidad como creación de expectativas y motivaciones personales, profesionales a través de la creación de actitudes y conocimiento. Se denomina Carrera profesional a los planes y programas, que contienen diversos elementos como perfiles, fundamentos, objetivos, estrategias, contenidos, medios para preparar a 
los profesionistas, en conocimientos, habilidades y actitudes, para cumplir determinados roles y funciones en la sociedad. Las carreras profesionales están divididas en especialidades, que se dan en función a la gran división del trabajo, y desarrollo de la ciencia y la tecnología.

Por su parte, (Díaz, Fert y otros.2000) propone una metodología que comprende cuatro etapas: Fundamentación de la Carrera Profesional, Elaboración del Perfil Profesional, Organización y Estructuración Curricular y Evaluación continua del currículo. Las Áreas y dimensiones que comprende la formación del profesor, como proceso sistemático de preparación teórica y metodológica del profesor se organiza y estructura en áreas y dimensiones procurando que en cada una de ellas existe coherencia y pertinencia plena. La organización de los contenidos, competencias y objetivos en áreas, dimensiones, aspectos, dominios, etc. varía según los sistemas educativos de cada país, pudiendo adquirir diferentes nombres, pero con significados similares.

Mención no menos importante (Gimeno, José y Martín, Ricardo. 2015) quien organiza los contenidos y competencias de la Formación Profesional del profesorado en tres áreas: Área de Cultura General, Área de Especialización y Área Pedagógica. Formación general. El profesor, como factor fundamental en el proceso educativo del ser humano, requiere poseer una vasta cultura, demostrable en todo momento de su vida, frente a los demás y especialmente ante los alumnos. El profesor ha de ser un hombre culto para difundir cultura, que por no surgir en despliegue de pura naturaleza, reclama un esfuerzo tenso y una colaboración especial, específica. Igualmente, para impartir conocimientos y ampliar el ámbito del saber, que es por excelencia la función de una institución educativa, se desarrolla los valores intelectuales, también es necesario desglosar las dimensiones: Humanísticas, científicas y técnica. Formación especialización. Es aquel dominio donde se ubican las asignaturas y contenidos, que tienen como propósito brindar al estudiante de educación, los conocimientos propios de su especialidad, que les servirá para el desenvolvimiento en su práctica profesional. Con las asignaturas de esta área el futuro docente profundiza, amplía y actualiza los conocimientos respecto a la materia o especialidad que ha elegido.

El profesor si ha de ser un eficaz colaborador del aprendizaje, capaz de dominar las técnicas fundamentales, las fuentes de información y responder adecuadamente a las preguntas del alumno en su marcha difícil hacia la conquista del: saber, saber hacer y saber vivir, tiene que circunscribirse a un área determinada, necesita conocer en profundidad las materias que ha de impartir, superar con mucho cuidado el nivel de información de los respectivos programas escolares y estar al día en la corriente desbordada de las nuevas aportaciones que nos vienen de los cinco continentes. En esta área de formación profesional del docente, intervienen, fundamentándola teóricamente, diversas ciencias sociales derivados de la relación con la 
educación, que ayudan a comprender mejor, tanto al mismo proceso educativo, como a los elementos y factores que intervienen en el. Nos referimos a las llamadas ciencias de la educación, a los que deberían llamarse ciencias auxiliares de la educación. Estas son: La Psicología Educativa, la Antropología Educativa, la Sociología de la Educación, la Psicología Evolutiva, la Economía de la Educación, la Política Educativa y la Epistemología de la Educación. Para el efecto propone que la formación profesional del docente debe estructurarse y organizarse en cinco áreas: Área de conocimiento de formación general, Área de conocimiento de formación profesional, Área de Actividades, Área de tutoría y Área de investigación.

\section{MATERIALES Y MÉTODOS}

El estudio utilizó el método lógico deductivo consistente en la aplicación de los principios teóricos sobre la Gestión Educativa procurando medir la relación con la Calidad de Formación Docente. La población estaba conformada por los egresados de la carrera de Lengua y Literatura. La muestra estuvo conformada por 65 estudiantes de la especialidad de Lengua y Literatura, 20 docentes del Instituto Superior Pedagógico Público “Gran Pajatén”.

Se utilizó el análisis de los diversos documentario, entrevista y cuestionario sobre aspectos de la gestión educativa en las dimensiones de gestión institucional, gestión administrativa, Gestión curricular. En cuanto a la formación profesional se tomó en cuenta las siguientes dimensiones: formación general, básica y especializada. La identificación de la relación a través de la correlación de Pearson.

\section{RESULTADOS Y DISCUSIÓN}

\section{Gestión Educativa}

\section{Gestión institucional}

Respecto a los resultados de la dimensión institucional sobre las decisiones políticas del Instituto Superior Pedagógico "Gran Pajatén” están orientadas a lograr su desarrollo institucional; (70\%) dijeron que $\mathrm{Si} ;(20 \%)$ dijeron parcialmente y (10\%) dijeron que No, están orientadas a lograr su desarrollo institucional. En relación a los aspectos que priorizan las decisiones de políticas de la institución (65\%) dijeron que solo priorizan lo administrativo; (30\%) dijeron que ambos aspectos y $(5 \%)$ priorizan lo académico. En cuanto a la priorización de política institucional manifestaron de la siguiente manera (60\%) que se prioriza lo administrativo; (30\%) que son los intereses particulares y (10\%) que la política institucional prioriza lo académico. En relación a las coordinaciones que realiza el Institución con otras carreras indicaron que (30\%) que se dan principalmente con propósitos académicos; (55\%) con propósitos administrativo; (5\%) con 
propósitos de desarrollo de proyectos; (5\%) con propósitos de extensión estudiantil y (5\%) con propósitos político y personal.

\section{Gestión administrativa}

\section{Planificación}

En cuanto si realizan planificación estratégica en la institución se obtuvo como resultado que (45\%) que no realiza planificación estratégica; (30\%) que sí, pero deficientemente y (25\%) que sí, eficientemente realiza planificación estratégica.

Respecto a los documentos con los que cuenta el ISP se obtuvo la siguiente información (95\%) cuenta con PAT; (85\%) cuenta con el Plan Curricular por Especialidades; (95\%) cuenta con Plan de Desarrollo Estratégico; (90\%) cuenta con Reglamento Interno.

En relación si el instituto superior pedagógico elabora su diagnóstico estratégico, se obtuvo como resultado que (45\%) dijeron que si, deficientemente; (25\%) que sí, pero eficientemente y (30\%) dijeron que no elabora su diagnóstico estratégico.

En cuanto a la información recogida en el diagnóstico estratégico, se debate en la Asamblea de Docentes, de un total de 20 docentes respondieron de la siguiente manera:(60\%) respondieron que a veces; (35\%) que no y (5\%) respondió que sí.

Al respecto, si se expone ante el pleno de docentes el Plan de Desarrollo Estratégico y el Proyecto de Desarrollo Institucional, respondieron de la siguiente manera: (70\%) que no exponen ante el pleno de docentes; $(25 \%)$ que a veces y $(\%)$ respondió que si exponen ante el pleno de docentes.

\section{Proceso de organización}

En cuanto si se realiza la designación y distribución de los cargos de acuerdo al Reglamento o Estatuto de la institución, respondieron de la siguiente manera: (55\%) respondieron no; (40\%) que parcialmente y (5\%) respondió que sí.

Respecto a la organización y distribución del personal administrativo respondieron de la siguiente manera: (35\%) respondieron que es deficiente; (40\%) que es eficiente y (25\%) respondieron que es pésima.

La organización y distribución del personal docente por especialidad es: En relación a la organización y distribución de docentes por Especialidad respondieron de la siguiente manera: (55\%) respondieron que es deficiente; (35\%) que es eficiente y (10\%) respondió que es pésima. 
Si la actual forma de organizar a los alumnos permite que todos tengan sus aulas, los docentes manifestaron (5\%) respondieron deficientemente; $(5 \%)$ respondió que no y $(90 \%)$ respondió que sí.

\section{Proceso de dirección}

Del total de docentes, respecto a la forma de conducción y dirección del Área Académicos, respondieron de la siguiente manera: (10\%) respondieron que es arbitraria; (55\%) respondieron que es deficiente; (25\%) respondieron que es eficiente; (10\%) respondieron que es politizada.

En razón a las decisiones que se toman en el Instituto Superior Pedagógico "Gran Pajatén", respondieron de la siguiente manera: (50\%) respondieron que obedecen a los intereses políticos de los grupos de poder; (5\%) que obedecen a los intereses económicos de los grupos de poder y $(45 \%)$ respondieron que obedecen a los intereses institucionales de la institución.

\section{Proceso de Control}

Los docentes de la institución, en cuanto a si existe un proceso de control para medir la eficiencia y eficacia de los procesos de gestión en la institución, respondieron de la siguiente manera: (75\%) respondieron que no; (20\%) respondieron que es deficiente y (5\%) respondió que sí.

Del total de maestros encuestados, respecto a quien realiza el control de las actividades de gestión en el instituto, respondieron de la siguiente manera: (46\%) que no se realiza control; (45\%) que ninguno de los mencionados; (20\%) que el Dirección; (25\%) que los Jefes de Área; (10\%) respondieron ninguno de las comisiones y ninguno del consejo de estudiantes.

Respecto a los sistemas de control que hay en el Instituto Superior Pedagógico, respondieron de la siguiente manera: (70\%) respondieron el Sistema de Control de Información; (15\%) respondieron ninguno; (15\%) respondió el Sistema de Evaluación de Rendimiento y ninguno el Sistema de Control Financiero.

\section{Gestión curricular}

\section{Planificación Curricular}

En relación con el Plan Curricular actualizado, respondieron de la siguiente manera: (75\%) respondieron que no; $(25 \%)$ respondieron que está medianamente y ninguno respondió que sí. Se evalúa la pertinencia de los sílabos con el Plan Curricular:

Respecto a si se ha evaluado la pertinencia de los syllabus con el actual Plan Curricular de la Institución, respondieron de la siguiente manera: (50\%) que a veces, (30\%) que no y (20\%) que sí. 


\section{Respecto al contenido del Plan Curricular y sus propósitos.}

En cuanto al contenido del Plan Curricular y sus propósitos, respondieron de la siguiente manera: (45\%) respondieron que requiere de algunos reajustes; (35\%) respondieron que requiere de muchos reajustes; (15\%) respondieron que es pertinente y (5\%) respondió que no es pertinente.

En relación a si las comisiones académicas exponen sus planes y proyectos ante el pleno de docentes para su discusión, análisis y aprobación, respondieron de la siguiente manera: (65\%) respondieron que no; $(25 \%)$ respondieron que a veces y (10\%) respondieron que sí.

Al finalizar el año académico las comisiones exponen ante el pleno de docentes sus logros y dificultades, para que sean tomados en cuenta en los trabajos de planificación del siguiente año alegaron de la siguiente manera: $(90 \%)$ respondieron que no; $(5 \%)$ respondió que sí y a veces.

\section{Implementación Curricular}

En cuanto a que el personal docente se encuentra debidamente implementado y actualizado, alegaron un $(50 \%)$ respondieron que medianamente; $(40 \%)$ respondieron que no y (10\%) respondieron que sí.

En cuanto a si el número de aulas es proporcional al número de alumnos, respondieron de la siguiente manera: (100\%) respondieron que sí; ninguno respondió parcialmente y que sí.

Respecto a si la implementación del laboratorio respecto a los materiales y equipos, respondieron de la siguiente manera: $(60 \%)$ respondieron que es deficiente; $(35 \%)$ respondieron que es pésima y (5\%) respondió que es eficiente.

En relación a la implementación de la biblioteca, reconocieron de la siguiente manera: (90\%) respondieron que es deficiente; (10\%) respondió pésima y ninguno de eficiente.

En cuanto a la implementación del Centro de Cómputo, manifestaron de la siguiente manera: (75\%) que es deficiente; (25\%) que es eficiente y ninguno que es pésima.

\section{Organización Curricular}

Respecto a la organización de docentes en escuelas y departamentos, se determinó que (75\%) manifestaron que es deficiente; (25\%) respondió que es eficiente y ninguno respondió que es pésima.

La organización de alumnos por especialidad manifestaron (95\%) que es eficiente; (5\%) que es deficiente. 
En cuanto a la distribución y ubicación de las aulas, respondieron de la siguiente manera: (85\%) respondieron que es eficiente; (15\%) respondió que es eficiente y ninguno respondió que es pésima.

En referencia a la ubicación y diseño de los locales para la biblioteca y el centro de cómputo y laboratorio, respondieron de la siguiente manera: (85\%) dijeron que es adecuado; (15\%) respondieron inadecuado y ninguno respondió pésima.

Respecto a la organización y ubicación de las asignaturas por áreas, respondieron de la siguiente manera: $(85 \%)$ que es eficiente; $(15 \%)$ que es deficiente y que ninguno es pésima.

\section{Dirección Curricular}

Respecto a las decisiones que toman las autoridades en materia académica, manifestaron (55\%) que son deficientes; (30\%) que son eficientes y (15\%) que son pésima.

En cuanto a si las autoridades del Instituto Superior Pedagógico convocan a reuniones de trabajo permanentemente, respondieron de la siguiente manera: $(60 \%)$ que no; $(25 \%)$ que a veces y $(15 \%)$ que sí.

En cuanto a organización de eventos científicos y académicos para promover el mejoramiento de la calidad de formación profesional, alegaron (50\%) que a veces; (35\%) que no, (15\%) que sí.

Respecto a las decisiones que toma en cada carrera respondieron de la siguiente manera: (65\%) dijeron que son inadecuadas; $(25 \%)$ respondió que son adecuadas y pésimas (10\%).

\section{Ejecución curricular}

En cuanto si los syllabus se elaboran en directa relación con el Plan Curricular, alegaron de la siguiente manera: (50\%) que a veces; (35\%) que sí y (15\%) que no.

Respecto si las clases se desarrollan de acuerdo a lo previsto en los syllabus, alegaron de la siguiente manera: (70\%) que sí; (20\%) que a veces y (10\%) que no.

En relación a las técnicas que más usan en el desarrollo de sus clases, manifestaron de la siguiente manera: (25\%) que es la técnica de debate dirigido; (20\%) que es exposiciones; (20\%) que es seminario; (15\%) que es dinámica de grupos; (15\%) que es lluvia de ideas; (5\%) que es el dictado.

En relación a los medios y materiales didácticos que utilizan en clase con mayor frecuencia, respondieron de la siguiente manera: (60\%) el papelógrafo; (25\%) el proyector; (15\%) pizarra y plumón; (10\%) los libro; (10\%) las separatas; (10\%) la TV y el VHS. 
Respecto a los métodos que emplea en el desarrollo de sus clases, respondieron de la siguiente manera: (100\%) que emplean los métodos mixtos y ninguno respondió, que sean los métodos activos ni pasivos

\section{Evaluación curricular}

Respecto a la clase de evaluación que aplica, respondieron de la siguiente manera: (65\%) la heteroevaluación; (20\%) la autoevaluación y (15\%) la coevaluación.

En relación a los ítems que más se emplean en la evaluación, respondieron de la siguiente manera: (50\%) que son los objetivos impresos y (50\%) que son los de ensayo o desarrollo.

Respecto a si se realiza la evaluación del Plan Curricular, respondieron que el (40\%) dijeron que sí; (35\%) parcialmente y (25\%) que no.

En conclusión la Gestión educativa se encuentra en una categoría regular.

Tabla 1: Resumen gestión educativa y sus indicadores

\begin{tabular}{|l|r|r|r|r|}
\hline \multicolumn{1}{|c|}{$\begin{array}{c}\text { VARIABLES E } \\
\text { INDICADORES }\end{array}$} & $\mathrm{N}$ & Media & Desv. típ. & \multicolumn{2}{|c|}{ Varianza } \\
\hline Gestión educativa & $\mathbf{2 0}$ & $\mathbf{1 , 9 3}$ & $\mathbf{, 6 9 1}$ & $\mathbf{, 4 7 8}$ \\
\cline { 1 - 5 } Gestión institucional & 20 & 1,77 &, 626 &, 392 \\
\hline $\begin{array}{l}\text { Gestión } \\
\text { administrativa }\end{array}$ & 20 & 1,73 &, 785 &, 616 \\
\hline $\begin{array}{l}\text { Gestión } \\
\text { curricular }\end{array}$ & 20 & 1,93 &, 691 &, 478 \\
\hline N válido (según lista) & 20 & & & \\
\hline
\end{tabular}

\section{Fuente: Elaboración propia}

En la presente tabla1 de Gestión Educativa y sus indicadores: el Indicador Gestión Institucional obtuvo una Media de 1,77, Gestión Administrativa de 1,73 y Gestión Curricular de 1,93. Siendo la media de la Variable Gestión Educativa de 1,93. Frente a la escala valorativa se encuentra en el rango de 11-14 de categoría regular

Formación Profesional: Formación general

En el área de Formación General de la especialidad de Lengua y Literatura a través de la revisión documentaria se pudo obtener la siguiente información de notas: (36,9\%) entre 13 y 14; 15 $(23,1 \%)$ entre 11 y $12 ;(16,9 \%)$ entre 15 y $16 ;(13,8 \%)$ entre 17 y $18 ;(3,1 \%)$ entre $19-20 ;(7.2 \%)$. Siendo la media 13,92 que es igual a 14.Lo es que aceptable y ubicado en la categoría de regular. 


\section{Formación Profesional Básica}

En el área de Formación Profesional Básica los resultados de las notas observadas fueron las siguientes: $(33,8 \%)$ entre 13 y $14 ;(27,7 \%)$ entre 15 y $16 ;(13,8 \%)$ entre 17 y $18 ;(10,8 \%)$ entre 11 y 12; $(9,2 \%)$ entre 07 y 08; (4,6\%) entre 09 y 10. Siendo la media de 13,63. Categorizada de regular el resultado.

Formación Profesional Especializada

En el área de Formación Profesional Especializada se obtuvieron las siguientes notas: (36,9\%) entre 15 y $16 ;(27,7 \%)$ entre 13 y $14 ;(18,5 \%)$ entre 17 y $18 ;(7,7 \%)$ entre 11 y 12,19 y20; $(1,5 \%)$ entre 09 y 10 . Siendo la media 15,23 , que es igual a15. Establecido el resultado en categoría de bueno.

Por lo tanto, en cuanto a la Formación Profesional y sus indicadores: el indicador Formación General tiene un promedio de 13,92, Formación Básica de 13,63 y Formación Especializada de 15,23. Encontrándose en una categoría regular.

Tabla 2: Resumen formación profesional y sus indicadores

\begin{tabular}{|l|r|r|r|r|}
\hline \multicolumn{1}{|c|}{$\begin{array}{r}\text { VARIABLES E } \\
\text { INDICADORES }\end{array}$} & $\mathrm{N}$ & Media & Desv. típ. & Varianza \\
\hline $\begin{array}{l}\text { FORMACIÓN } \\
\text { PROFESIONAL (Vd) }\end{array}$ & $\mathbf{6 5}$ & $\mathbf{1 3 , 6 5}$ & $\mathbf{2 , 4 8 0}$ & $\mathbf{6 , 1 4 9}$ \\
\hline Formación general & 65 & 13,92 & 2,878 & 8,280 \\
\hline Formación básica & 65 & 13,63 & 2,272 & 5,160 \\
\hline Formación especializada & 65 & 15,23 & 1,87 & 3,525 \\
\hline N válido (según lista) & 65 & & & \\
\hline
\end{tabular}

Fuente: Elaboración propia

\section{CONCLUSIÓN O CONSIDERACIONES FINALES}

Como resultado del trabajo de investigación concluimos que existe una relación alta y significativa entre la Gestión Educativa de nivel regular y la Calidad de Formación Profesional de nivel, también regular, con una correlación de 73,1\%. Se explica en el sentido de que se ha obtenido como puntaje de Gestión Educativa una media de 1,93 lo que en su escala valorativa equivale a "regular", y como promedio de Calidad de Formación Profesional una nota de 13,65 que en su escala valorativa equivale a "regular". 
En la presente investigación se ha determinado que existe una relación directa y positiva entre la Gestión Institucional y la calidad de Formación Profesional que se realiza en el Instituto Superior Pedagógico “Gran Pajatén”, con una correlación de 68.4\%. La relación está referida a que se ha obtenido como puntaje en Gestión Institucional una media de 1,77, lo que en su escala valorativa equivale a "regular", y como promedio de Calidad de Formación Profesional la nota de 13.65, que en su escala valorativa equivale también a "regular".

Podemos explicar que existe una relación directa entre la Gestión Administrativa y la Calidad de Formación Profesional que se realiza en el Instituto Superior Pedagógico “Gran Pajatén”, debido a que el índice de correlación de 81,8\%, lo que significa que la relación es alta y positiva. La relación está determinada a que se ha obtenido como puntaje de Gestión Administrativa una media de 1,73 lo que en su escala valorativa equivale a "regular", y como promedio de Calidad de Formación profesional se ha obtenido la nota de 13.65 , que en su escala valorativa equivale también a "regular".

Se ha llegado a conocer que la Gestión Curricular tiene relación directa y positiva con la Calidad de Formación Profesional que se realiza en el Instituto Superior Pedagógico “Gran Pajatén”, siendo el índice de correlación al 71,1\%, lo que significa que es una relación alta positiva.

La relación está referida a que se ha obtenido como puntaje de Gestión Curricular una media de 1,93 lo que en su escala valorativa equivale a "regular", y como promedio de Calidad de Formación profesional se ha obtenido la nota de 13.65, que en su escala valorativa equivale también a "regular". 


\section{LISTA DE REFERENCIAS}

Alvarado, O.(1998) Política Educativa. Conceptos, Reflexiones y Propuestas. Editorial Vallejiana, Universidad César Vallejo. Trujillo Perú.

Angulo, G. (2012). Las concepciones sobre el docente o diferentes maneras de concebir el ejercicio de la docencia. Revista de Investigación, 36 (75), 11-32.

Bltad, J. (995) Formación del Profesor y Calidad de la Educación.

Bustamante, A. (2006). Educación, compromiso social y formación docente.

Revista Iberoamericana de Educación, 37(4), 1-8.

https://doi.org/10.35362/rie3742694.

Carrasco, S. O. (1995) Metodología de la Investigación Científica. Aplicación en educación y otras ciencias sociales. Primera Edición. Editorial San Marcos. Lima Perú.

Chiavenato, I. (1999) Introducción a la Teoría General de la Administración. Quinta Edición, Editorial Mc Graw Hill. Colombia.

Cifali, M. (2005). Enfoque clínico, formación y escritura. En L. Paquay (Coord.), La formación profesional del maestro. Estrategias y competencias (pp. 170-196). México: Fondo de Cultura Económica.

Colección Gerencia de Centros Educativos. Lima Perú.

Cruz, J. y Taborda, J. (2014). Hacia un giro fenomenológico en la pedagogía; el asunto de la experiencia en la pedagogía. Folios, 39, 161171.https://doi.org/10.17227/01234870.39folio 161.171

De La Torre, E. y Navarro De Anda, R. (1982) Metodología de la Investigación. Editorial Mc Graw Hill. México.

Delors, J. (1996) La Educación Encierra un Tesoro. Editorial Santillana. Ediciones

Díaz, V. (2006). Formación docente, práctica pedagógica y saber pedagógico. Revista de Educación Laurus, 12 (número extraordinario), 88-103.

Farro, F.)2000) Planeamiento Estratégico para Instituciones de Calidad. CPC, Lima Perú. 
Gallegos, J. (2004) Gestión educativa en el proceso de descentralización. Editorial San Marcos. Lima Perú.

Gomero, G. y Moreno J. (1997) Procesos de la Investigación Científica. Fakir Editores. Lima Perú.

Hermida, J. y otros. (1996) Administración Estratégica. Ediciones Macchi. Buenos Aires Hernández, G. (2003) Gestión y Administración Educativa (Antología). Centro de Extensión Universitaria y Proyección Social, UNMSM. Lima Perú.

Hernández S., Fernández C. y Baptista L. (1999) Metodología de la Investigación Científica. Segunda Edición. Editorial Mc Graw Hill México.

Hit, I y Hoskinsson. (1999). Administración Estratégica. Tercera Edición.

Internacional Thomson Editores. México.

Imbernon, F. (1994) La formación del Profesorado. Cuaderno de Pedagogía. Ediciones PAIDOS. Barcelona España.

Koontz, H. y Weihrich, H. (1997) Administración: una perspectiva global. Décima. Editorial Mc Graw Hill Interamericana México.

Koth, M. (1996) Estrategias para a la Gestión Educativa. Primera Edición. Fondo de Desarrollo Editorial. Universidad de Lima. Perú.

Kravetz, D. (1990) La Revolución de los Recursos Humanos. Una nueva dimensión de la gestión empresarial. Ediciones Deusto. Bilbao España.

Leboterf, G. y otros. (1993) Cómo Gestión la Calidad de la Formación Ediciones Gestión 2000. Barcelona España.

Lepeley, M. (2001) Gestión y Calidad en Educación. Un modelo de evaluación. Editorial Graw Mc Hill. Interamericana. Santiago de Chile.

Loaiza, Y. (2018). Los maestros y la pedagogía. Revista Latinoamericana de Estudios Educativos,14(1).http://190.15.17.25/latinoamericana/downloads/Latinoamerica na14(1)_1.pdf.

López, F. (1994) La Gestión de Calidad en Educación. La Muralla. Madrid España. 
Ministerio De Educación. (2016) Manual para el Docente. Programa de Formación Continua de Docentes en Servicio. Educación Secundaria. Lima Perú.

Puelles, M y otros. (1986) Elementos de la Administración Educativa Educación. 2da, Edición. Ministerio de Educación. Madrid España.

Quinn, R. y otros. (1995) Maestría en Gestión de Organizaciones. Un modelo operativo de competencias. Ediciones Díaz Santos, S. A. Madrid España.

Porter, M. (1998) Ser competitivos. Nuevas aportaciones y conclusiones. Ediciones DEUSTO S.A. España.

Thompson, J. R. y Strickland III, A. J. (1995) Dirección y administración estratégica. Editorial IRWIN. Madrid España.

Ugarte, M. y otros. (2003) Estudios en ciencias administrativas. Año 1, Volumen I, N ${ }^{\circ}$ 01. Escuela de Postgrado de la F.C.A de la Universidad Nacional Mayor de San Marcos. Lima Perú. 